\title{
Development of an Artificial Neural Network Model and Comparison with Nomogram for Prediction of Pathological Complete Response After Neoadjuvant Chemotherapy in Breast Cancer
}

Ji-Jung Jung

Seoul National University Bundang Hospital

\section{Eunyoung Kang}

Seoul National University Bundang Hospital

\section{Eun-Kyu Kim}

Seoul National University Bundang Hospital Jee Hyun Kim

Seoul National University Bundang Hospital

Se Hyun Kim

Seoul National University Bundang Hospital

Koung Jin Suh

Seoul National University Bundang Hospital

Sun Mi Kim

Seoul National University Bundang Hospital

Mijung Jang

Seoul National University Bundang Hospital

\section{Bo La Yun}

Seoul National University Bundang Hospital

\section{So Yeon Park}

Seoul National University Bundang Hospital

Hee-Chul Shin ( $\nabla$ dradam77@naver.com )

Seoul National University Bundang Hospital

\section{Research Article}

Keywords: Breast cancer, Machine learning, Neural network, Nomogram, Neoadjuvant chemotherapy, Complete response

Posted Date: June 17th, 2021 
DOl: https://doi.org/10.21203/rs.3.rs-609628/v1

License: (c) (1) This work is licensed under a Creative Commons Attribution 4.0 International License. Read Full License 


\section{Abstract}

Identifying breast cancer patients who may benefit from neoadjuvant chemotherapy will facilitate personalized treatment regarding chemotherapy and surgery. In our work, we developed two predictive models, nomogram and a machine learning model based on artificial neural network (ANN), to anticipate pathological complete response (pCR) after neoadjuvant chemotherapy (NAC) in breast cancer. We demonstrated that high level of estrogen receptor (ER) positivity, positive human epidermal growth factor receptor 2 (HER2) status, complete response on magnetic resonance imaging (MRI), abnormal CEA level after NAC, and abnormal CA15-3 level after NAC were significant predictors of pCR. A nomogram and ANN model trained to predict PCR were developed using these five predictors. The performance of the two models were tested using a fully independent test set. Validation test showed the area under the receiver operating characteristic curve (AUC) of 0.789 (95\% confidence interval (Cl), 0.707-0.871) for the nomogram and $0.876(95 \% \mathrm{Cl}, 0.808-0.943)$ for the ANN model. Both models showed excellent performance, but the ANN model performed better in terms of accuracy and discrimination. Machinelearning algorithms hold promise in medical application and provide better prediction than nomogram.

\section{Introduction}

Neoadjuvant chemotherapy (NAC) has become the standard treatment for locally advanced or unresectable breast cancer. NAC allows unresectable tumor to become operable while downstaging operable tumor to allow breast conservation ${ }^{1,2}$. Pathologic complete response (pCR) of breast cancer after NAC is a well-known outcome that correlates with better prognosis, higher event-free survival and overall survival ${ }^{3,4}$.

Studies have tried to find useful molecular, clinical or radiologic predictors of pCR to NAC. Imaging studies, such as magnetic resonance imaging (MRI) and sonography are often used to demonstrate change in tumor extent and predict response to NAC ${ }^{5}$. Human epidermal growth factor receptor 2 (HER2) overexpression and lack of estrogen receptor (ER) and progesterone receptor (PR) expression have been identified as potential predictors of pCR ${ }^{6,7}$. High level of serum tumor marker CA $15-3$ was predictive of poor pathological response ${ }^{8}$. Therefore, development of a method to combine multiple prognostic factors to predict a PCR would allow patient stratification and more appropriate treatment regimens. Patients with high probability of complete response could benefit from early resection, while nonresponders to NAC could be further treated with hormonal therapy or alternative regimens.

Nomogram is one of the most popular statistical tool to provide overall probability of certain outcome and several nomograms were developed to predict residual tumor size or pathologic response following NAC ${ }^{9-12}$. However, a nomogram assumes a linear relationship between predictors and outcome, and a transformation is needed when the relationship is non-linear. Alternatively, a variety of machine learning algorithms, such as decision trees and artificial neural network (ANN), have been recently applied in cancer prognosis ${ }^{13,14}$. Machine learning allows inclusion of larger number of input variables and complex data with non-linear relationship can be handled more comprehensively. Moreover, some 
algorithms even perform feature selection as part of their overall operation, and this reduces the risk of omitting some covariates as required in many predictive models.

In this study, we developed a machine learning method based on ANN algorithm to predict pCR after NAC in patients with advanced breast cancer. Moreover, we built a nomogram with the same dataset and further compared predictive accuracy of the two models for breast cancer prognosis.

\section{Methods}

\section{Patients}

Patients with histology-proven invasive breast carcinoma and who met the selection criteria were included in this study. The inclusion criteria were patients who (1) received breast conserving surgery or total mastectomy between January 2017 and December 2019; (2) received NAC prior to surgical resection. The exclusion criteria were patients who were diagnosed with recurred tumor. In total, 359 patients were included and their clinical data from electronic medical system were obtained, retrospectively. All methods were carried out in accordance with relevant guidelines and regulations. The Institutional Review Board (IRB) of Seoul National University Bundang Hospital waived the need of informed consent from patients because this was retrospective study based on medical records and had minimal risk to included patients even if the informed consent was waived. This study was approved by the IRB of Seoul National University Bundang Hospital (IRB No. B-2105/682-102).

\section{Statistical Analysis}

Univariate analyses were performed to determine whether clinicopathologic characteristics including age, body mean index, multiplicity, palpability, family history, clinical tumor stage, clinical node stage, histologic grade, nuclear grade, ER, PR, HER2 status, size of tumor at MRI, tumor-associated antigens (carcinoembryonic antigen (CEA) and carbohydrate antigen 15-3 (CA15-3)) were associated with pCR after NAC. Continuous variables were examined by student's t-test and presented as mean and standard deviation. Categorial variables were examined by chi-square test and expressed as number and percentage. For multivariate analyses, only variables with a significant association with $\mathrm{PCR}$ in the univariate analyses were used. Backward elimination method was carried out using logistic regression analyses and predictors were expressed by the odds ratio (OR) and $95 \%$ confidence interval $(\mathrm{Cl})$. Before developing predictive models, $\mathrm{PCR}$ group and non-pCR group were balanced using Synthetic Minority Over-sampling Technique (SMOTE) ${ }^{15}$. The balanced dataset was randomly split into training and test sets with a ratio of 7:3. R software version 3.6.3 (The R Foundation for Statistical Computing, Vienna, Austria) was used for statistical analyses.

\section{Model Construction and Evaluation}


Predictive models were built using status of $\mathrm{pCR}$ as the outcome variable. The training set was used to build predictive models and test set was used to assess the models. Significant variables from multivariate logistic regression analysis were included as predictors. For nomogram, continuous variables with a wide range, i.e. CA15-3 and CEA, were converted into categorical variable because range observed in the training set may not fully represent real world data. For machine learning model, a widely used neural network package, neuralnet, was used with multiple hidden layers and nodes. Discrimination power of each model was quantified by the area under the receiver-operating characteristics curve (AUC).

\section{Results}

\section{Patient Characteristics}

A total of 359 breast cancer patients who received NAC prior to surgical resection were included in this study. Patient demographics and clinical features are summarized in Table 1. Among the patients, 106 (29.5\%) patients achieved pCR after NAC. Univariate analyses showed that negative ER (74.5 vs. $47.0 \%, p$ $<0.001$ ), negative PR ( 90.6 vs. $64.0 \%, p<0.001$ ), positive HER2 ( 66.7 vs. $30.6 \%, p<0.001$ ), smaller tumor size on initial MRI (3.6 vs. $4.2 \mathrm{~cm}, p=0.005)$, higher level of pre-NAC Ki-67 (43.0 vs. $36.7 \%, p=0.004)$, lower level of pre-NAC CA15-3 (11.1 vs. $16.2 \mathrm{ng} / \mathrm{mL}, p=0.030)$, lower level of pre-NAC CEA (2.0 vs. 2.8 $\mathrm{ng} / \mathrm{mL}, p=0.030)$, complete response on post-NAC MRI (67.0 vs. $13.4 \%, p<0.001)$, lower level of postNAC CA15-3 (14.4 vs. $16.7 \mathrm{ng} / \mathrm{mL}, p=0.004)$, and lower level of post-NAC CEA $(2.3 \mathrm{vs.} 3.0 \mathrm{ng} / \mathrm{mL}, p=$ 0.001 ) were independent predictors of $\mathrm{pCR}$. 
Table 1

Clinical features and their correlation with $\mathrm{pCR}$

\begin{tabular}{|c|c|c|c|c|}
\hline & Total & $\begin{array}{l}\text { pCR }(n= \\
106)\end{array}$ & $\begin{array}{l}\text { Non-pCR }(n= \\
253)\end{array}$ & $p$ \\
\hline Age & $\begin{array}{l}49.5 \pm \\
10.3\end{array}$ & $50.3 \pm 10.1$ & $49.2 \pm 10.4$ & 0.338 \\
\hline BMI & $23.8 \pm 3.7$ & $23.4 \pm 3.0$ & $24.0 \pm 3.9$ & 0.175 \\
\hline Multiplicity & & & & 0.306 \\
\hline No & $\begin{array}{l}321 \\
(89.4 \%)\end{array}$ & 98 (92.5\%) & $223(88.1 \%)$ & \\
\hline Yes & $38(10.6 \%)$ & $8(7.5 \%)$ & $30(11.9 \%)$ & \\
\hline Palpability & & & & 1.000 \\
\hline No & 79 (22.0\%) & $23(21.7 \%)$ & $56(22.1 \%)$ & \\
\hline Yes & $\begin{array}{l}280 \\
(78.0 \%)\end{array}$ & $83(78.3 \%)$ & 197 (77.9\%) & \\
\hline Family history & & & & 1.000 \\
\hline No & $\begin{array}{l}287 \\
(83.4 \%)\end{array}$ & $84(83.2 \%)$ & $203(83.5 \%)$ & \\
\hline Yes & $57(16.6 \%)$ & $17(16.8 \%)$ & $40(16.5 \%)$ & \\
\hline Clinical tumor stage & & & & 0.163 \\
\hline cT1 & $29(8.1 \%)$ & $11(10.4 \%)$ & $18(7.1 \%)$ & \\
\hline cT2 & $\begin{array}{l}227 \\
(63.2 \%)\end{array}$ & $73(68.9 \%)$ & $154(60.9 \%)$ & \\
\hline cT3 & $77(21.4 \%)$ & $17(16.0 \%)$ & $60(23.7 \%)$ & \\
\hline cT4 & $26(7.2 \%)$ & $5(4.7 \%)$ & $21(8.3 \%)$ & \\
\hline Clinical node stage & & & & 0.720 \\
\hline cNO & $85(23.7 \%)$ & $25(23.6 \%)$ & $60(23.7 \%)$ & \\
\hline cN1 & $\begin{array}{l}158 \\
(44.0 \%)\end{array}$ & 47 (44.3\%) & 111 (43.9\%) & \\
\hline $\mathrm{cN} 2$ & $62(17.3 \%)$ & $21(19.8 \%)$ & $41(16.2 \%)$ & \\
\hline $\mathrm{cN} 3$ & 54 (15.0\%) & $13(12.3 \%)$ & 41 (16.2\%) & \\
\hline
\end{tabular}

pCR: pathologic complete response, BMI: body mass index, ER: estrogen receptor, PR: progesterone receptor, HER2: human epidermal growth factor receptor 2, NAC: neoadjuvant chemotherapy, MRI: magnetic resonance image 


\begin{tabular}{|c|c|c|c|c|}
\hline & Total & $\begin{array}{l}\mathrm{pCR}(\mathrm{n}= \\
106)\end{array}$ & $\begin{array}{l}\text { Non-pCR }(n= \\
253)\end{array}$ & $p$ \\
\hline Histologic grade & & & & 0.059 \\
\hline G1 & $7(1.9 \%)$ & $1(0.9 \%)$ & $6(2.4 \%)$ & \\
\hline G2 & $\begin{array}{l}129 \\
(35.9 \%)\end{array}$ & $29(27.4 \%)$ & $100(39.5 \%)$ & \\
\hline G3 & $\begin{array}{l}216 \\
(60.2 \%)\end{array}$ & 75 (70.8\%) & 141 (55.7\%) & \\
\hline Missing & $7(1.9 \%)$ & $1(0.9 \%)$ & $6(2.4 \%)$ & \\
\hline Nuclear grade & & & & 0.083 \\
\hline G1 & $2(0.6 \%)$ & $0(0.0 \%)$ & $2(0.8 \%)$ & \\
\hline G2 & $\begin{array}{l}100 \\
(27.9 \%)\end{array}$ & $21(19.8 \%)$ & 79 (31.2\%) & \\
\hline G3 & $\begin{array}{l}251 \\
(69.9 \%)\end{array}$ & $84(79.2 \%)$ & 167 (66.0\%) & \\
\hline Missing & $6(1.7 \%)$ & $1(0.9 \%)$ & $5(2.0 \%)$ & \\
\hline ER status & & & & $\begin{array}{l}<.001 \\
0.001\end{array}$ \\
\hline High positive & $\begin{array}{l}133 \\
(37.0 \%)\end{array}$ & $15(14.2 \%)$ & $118(46.6 \%)$ & \\
\hline Low positive & $21(5.8 \%)$ & $8(7.5 \%)$ & $13(5.1 \%)$ & \\
\hline Negative & $\begin{array}{l}198 \\
(55.2 \%)\end{array}$ & 79 (74.5\%) & $119(47.0 \%)$ & \\
\hline Missing & $7(1.9 \%)$ & $4(3.8 \%)$ & $3(1.2 \%)$ & \\
\hline PR status & & & & $<.001$ \\
\hline Positive & $\begin{array}{l}101 \\
(28.1 \%)\end{array}$ & $10(9.4 \%)$ & 91 (36.0\%) & \\
\hline Negative & $\begin{array}{l}258 \\
(71.9 \%)\end{array}$ & $96(90.6 \%)$ & $162(64.0 \%)$ & \\
\hline HER2 status & & & & $<.001$ \\
\hline Positive & $\begin{array}{l}146 \\
(41.4 \%)\end{array}$ & 70 (66.7\%) & 76 (30.6\%) & \\
\hline $\begin{array}{l}\text { pCR: pathologic } \\
\text { receptor, HER2: hy } \\
\text { magnetic resonar }\end{array}$ & $\begin{array}{l}\text { y mass inc } \\
\text { or receptor }\end{array}$ & $\begin{array}{l}\text { ER: estrog } \\
\text { NAC: neoac }\end{array}$ & $\begin{array}{l}\text { ceptor, PR: pro } \\
\text { nt chemothera }\end{array}$ & $\begin{array}{l}\text { rone } \\
\text { IRI: }\end{array}$ \\
\hline
\end{tabular}




\begin{tabular}{|c|c|c|c|c|}
\hline & Total & $\begin{array}{l}\text { PCR }(n= \\
106)\end{array}$ & $\begin{array}{l}\text { Non-pCR }(n= \\
253)\end{array}$ & $p$ \\
\hline Negative & $\begin{array}{l}207 \\
(58.6 \%)\end{array}$ & 35 (33.3\%) & $172(69.4 \%)$ & \\
\hline $\begin{array}{l}\text { Longest tumor diameter before NAC, MRI } \\
(\mathrm{cm})\end{array}$ & $4.0 \pm 2.1$ & $3.6 \pm 1.8$ & $4.2 \pm 2.2$ & 0.005 \\
\hline Pre-NAC Ki-67 (\%) & $\begin{array}{l}38.6 \pm \\
18.8\end{array}$ & $43.0 \pm 19.4$ & $36.7 \pm 18.2$ & 0.004 \\
\hline Pre-NAC CA15-3 (U/mL) & $\begin{array}{l}14.7 \pm \\
15.5\end{array}$ & $11.1 \pm 5.5$ & $16.2 \pm 17.8$ & $<.001$ \\
\hline Pre-NAC CA15-3 & & & & 0.012 \\
\hline Normal $(<25)$ & $\begin{array}{l}327 \\
(91.1 \%)\end{array}$ & $100(94.3 \%)$ & $227(89.7 \%)$ & \\
\hline Abnormal ( $\geq 25$ ) & $28(7.8 \%)$ & $3(2.8 \%)$ & $25(9.9 \%)$ & \\
\hline Missing & $4(1.1 \%)$ & $3(2.8 \%)$ & $1(0.4 \%)$ & \\
\hline Pre-NAC CEA (ng/mL) & $2.6 \pm 3.7$ & $2.0 \pm 2.5$ & $2.8 \pm 4.1$ & 0.030 \\
\hline Pre-NAC CEA & & & & 0.056 \\
\hline Normal $(<5)$ & $\begin{array}{l}324 \\
(90.3 \%)\end{array}$ & $98(92.5 \%)$ & $226(89.3 \%)$ & \\
\hline Abnormal ( $\geq 5$ ) & $28(7.8 \%)$ & $4(3.8 \%)$ & $24(9.5 \%)$ & \\
\hline Missing & $7(1.9 \%)$ & $4(3.8 \%)$ & $3(1.2 \%)$ & \\
\hline $\begin{array}{l}\text { Longest tumor diameter after NAC, MRI } \\
(\mathrm{cm})\end{array}$ & $1.7 \pm 1.8$ & $0.6 \pm 1.3$ & $2.2 \pm 1.8$ & $<.001$ \\
\hline Complete response on MRI & & & & $\begin{array}{l}< \\
0.001\end{array}$ \\
\hline No & $\begin{array}{l}248 \\
(69.1 \%)\end{array}$ & $34(32.1 \%)$ & $214(84.6 \%)$ & \\
\hline Yes & $\begin{array}{l}105 \\
(29.2 \%)\end{array}$ & $71(67.0 \%)$ & $34(13.4 \%)$ & \\
\hline Missing & $6(1.7 \%)$ & $1(0.9 \%)$ & $5(2.0 \%)$ & \\
\hline Post-NAC CA15-3 (U/mL) & $16.0 \pm 8.1$ & $14.4 \pm 5.6$ & $16.7 \pm 8.8$ & 0.004 \\
\hline Post-NAC CA15-3 & & & & 0.016 \\
\hline
\end{tabular}

pCR: pathologic complete response, BMI: body mass index, ER: estrogen receptor, PR: progesterone receptor, HER2: human epidermal growth factor receptor 2, NAC: neoadjuvant chemotherapy, MRI: magnetic resonance image 


\begin{tabular}{|c|c|c|c|c|}
\hline & Total & $\begin{array}{l}\mathrm{pCR}(\mathrm{n}= \\
106)\end{array}$ & $\begin{array}{l}\text { Non-pCR }(n= \\
253)\end{array}$ & $p$ \\
\hline Normal (<25) & $\begin{array}{l}327 \\
(91.1 \%)\end{array}$ & $103(97.2 \%)$ & $224(88.5 \%)$ & \\
\hline Abnormal ( $\geq 25$ ) & $32(8.9 \%)$ & $3(2.8 \%)$ & $29(11.5 \%)$ & \\
\hline Post-NAC CEA (ng/mL) & $2.8 \pm 2.7$ & $2.3 \pm 1.3$ & $3.0 \pm 3.1$ & 0.001 \\
\hline Post-NAC CEA & & & & 0.039 \\
\hline Normal $(<5)$ & $\begin{array}{l}324 \\
(90.3 \%)\end{array}$ & $102(96.2 \%)$ & $222(87.7 \%)$ & \\
\hline Abnormal ( $\geq 5$ ) & $31(8.6 \%)$ & $3(2.8 \%)$ & $28(11.1 \%)$ & \\
\hline Missing & $4(1.1 \%)$ & $1(0.9 \%)$ & $3(1.2 \%)$ & \\
\hline
\end{tabular}

\section{Significant Risk Factors Associated with pCR after NAC}

Multivariate logistic regression analysis showed that factors which significantly affected possibility of PCR were: high level of ER positivity (OR $0.84, p<0.001$ ), positive HER2 status (OR 1.25, $p<0.001$ ), complete response on post-NAC MRI (OR 1.62, $p<0.001$ ), abnormal CEA level after NAC (OR 0.86, $p=$ 0.051), and abnormal CA15-3 level after NAC (OR 0.87, $p=0.074)$ (Table 2). 
Table 2

Predictive factor for $\mathrm{pCR}$, estimated by univariate and multivariate logistic regression analyses

\begin{tabular}{|c|c|c|c|c|c|c|}
\hline & \multicolumn{3}{|c|}{ Univariate analysis } & \multicolumn{3}{|c|}{ Multivariate analysis } \\
\hline & OR & $95 \% \mathrm{Cl}$ & $\begin{array}{l}p \\
\text { value }\end{array}$ & OR & $95 \% \mathrm{Cl}$ & $\begin{array}{l}p \\
\text { value }\end{array}$ \\
\hline Age & 1.00 & $\begin{array}{l}1.00- \\
1.01\end{array}$ & 0.903 & & & \\
\hline BMI & 0.99 & $\begin{array}{l}0.98- \\
1.01\end{array}$ & 0.213 & & & \\
\hline Multiplicity (yes) & 0.89 & $\begin{array}{l}0.75- \\
1.06\end{array}$ & 0.193 & & & \\
\hline Palpability (yes) & 1.00 & $\begin{array}{l}0.88- \\
1.15\end{array}$ & 0.972 & & & \\
\hline Family history (yes) & 1.00 & $\begin{array}{l}0.87- \\
1.15\end{array}$ & 0.995 & & & \\
\hline \multicolumn{7}{|l|}{ Clinical tumor stage } \\
\hline cT1 & Ref & & & & & \\
\hline cT2 & 0.88 & $\begin{array}{l}0.72- \\
1.07\end{array}$ & 0.193 & & & \\
\hline cT3 & 0.84 & $\begin{array}{l}0.67- \\
1.05\end{array}$ & 0.132 & & & \\
\hline cT4 & 0.74 & $\begin{array}{l}0.56- \\
0.97\end{array}$ & 0.031 & & & \\
\hline \multicolumn{7}{|l|}{ Clinical node stage } \\
\hline cNO & Ref & & & & & \\
\hline $\mathrm{cN} 1$ & 0.90 & $\begin{array}{l}0.79- \\
1.03\end{array}$ & 0.143 & & & \\
\hline $\mathrm{cN} 2$ & 0.93 & $\begin{array}{l}0.79- \\
1.10\end{array}$ & 0.379 & & & \\
\hline cN3 & 0.80 & $\begin{array}{l}0.67- \\
0.95\end{array}$ & 0.012 & & & \\
\hline \multicolumn{7}{|l|}{ Histologic grade } \\
\hline G1 & Ref & & & & & \\
\hline G2 & 1.21 & $\begin{array}{l}0.83- \\
1.74\end{array}$ & 0.320 & & & \\
\hline G3 & 1.31 & $\begin{array}{l}0.91- \\
1.89\end{array}$ & 0.147 & & & \\
\hline
\end{tabular}


Nuclear grade

G1

G2

G3

ER status (Level)

Negative

Low positive

High positive

PR status (positive)

HER2 status (positive)

Longest tumor diameter before NAC, MRI (cm)

Pre-NAC Ki-67 (\%)

Pre-NAC CA15-3 (U/mL)

Pre-NAC CA15-3

Normal $(<25)$

Abnormal $(\geq 25)$

Pre-NAC CEA (ng/mL)

Pre-NAC CEA

Normal $(<5)$

Abnormal $(\geq 5)$

Longest tumor diameter after NAC, MRI (cm)
Ref

$\begin{array}{lll}1.31 & \begin{array}{l}0.67- \\ 2.58\end{array} & 0.429 \\ & 1.52 & 0.78- \\ & 2.97 & 0.220 \\ & & \end{array}$

Ref

0.98

$0.79-$

1.23

$0.75 \quad 0.67-$

0.83

0.77

$0.68-$

0.86

1.40

$1.27-$
1.55

$0.97 \quad 0.95-$

1.00

$\begin{array}{ll}1.00 & 1.00- \\ & 1.00\end{array}$

$\begin{array}{ll}0.99 \quad 0.99 \\ & 1.00\end{array}$
Ref

$\begin{array}{llll}0.892 & 0.98 & \begin{array}{l}0.82- \\ 1.17\end{array} & 0.809\end{array}$

$\begin{array}{llll}< & 0.82 & 0.75- & < \\ 0.001 & & 0.90 & 0.001\end{array}$

$<$

0.001

$\begin{array}{llll}< & 1.25 & 1.15- & < \\ 0.001 & & 1.36 & 0.001\end{array}$

0.048

0.295

0.001

Ref

$\begin{array}{lll}0.76 & \begin{array}{ll}0.62- \\ 0.93\end{array} & 0.008\end{array}$

$0.99 \quad 0.97-$

0.078
Ref

$\begin{array}{lll}0.85 & \begin{array}{l}0.70- \\ 1.04\end{array} & 0.109 \\ 0.91 & \begin{array}{l}0.88- \\ 0.94\end{array} & < \\ & 0.001\end{array}$


Complete response on MRI

\begin{tabular}{|lcccccc|}
\hline No & Ref & \multicolumn{5}{c|}{ Ref } \\
Yes & 1.79 & $1.62-$ & $<$ & 1.63 & $1.48-$ & $<$ \\
Post-NAC CA15-3 (U/mL) & & 1.97 & 0.001 & & 1.79 & 0.001 \\
Post-NAC CA15-3 & 0.99 & $0.99-$ & 0.011 & & & \\
Normal $(<25)$ & & 1.00 & & & & \\
Abnormal $(\geq 25)$ & & & & & & \\
& Ref & & & Ref & & \\
Post-NAC CEA $(\mathrm{ng} / \mathrm{mL})$ & 0.75 & $0.62-$ & 0.004 & 0.86 & $0.74-$ & 0.051 \\
& & 0.91 & & & 1.00 & \\
Post-NAC CEA & 0.95 & $0.93-$ & 0.001 & & & \\
Normal $(<5)$ & & 0.98 & & & & \\
\hline Abnormal $(\geq 5)$ & & & & & & \\
& Ref & & & Ref & & \\
\hline
\end{tabular}

\section{Development and Validation of the Nomogram and the ANN Model}

Using the training set and five independent variables identified by the multivariate logistic regression analyses, a nomogram was developed to predict pCR after NAC (Fig. 1). Total point calculated by sum of the points for each variable is used to identify the probability of pCR after NAC. Similarly, we developed an ANN model (Fig. 2). First input layer consists of the five independent variables as used in the nomogram, and number of layers and nodes were determined based on systematic experimentation that worked best for our dataset. The prediction rate was best when hidden layers were set to three and each node to two.

The two prediction models were internally validated using the test set and receiver operating characteristic (ROC) curves are depicted in Fig. 3-4. The AUC of the machine learning model and nomogram were $0.876(95 \% \mathrm{Cl}, 0.808-0.943)$ and $0.789(95 \% \mathrm{Cl}, 0.707-0.871)$, respectively. Discriminative power of the machine learning model was higher than that of the nomogram.

\section{Discussion}

The present study developed two independent predictive models to predict pCR after NAC in breast cancer patients based on clinicopathologic indicators. Multivariate analysis showed that breast cancer with 
lower level of ER expression, HER2 positivity, complete response on post-NAC MRI, normal CEA and CA 15 - 3 levels after NAC were more likely to achieve a pCR after NAC. With an AUC of 0.789 [95\% Cl, 0.707$0.871]$ for the nomogram and 0.876 [95\% Cl, 0.808-0.943] for the ANN model, the two models both exhibited sufficient power to predict pCR after NAC in breast cancer, however, the ANN model outperformed nomogram in terms of accuracy and precision.

Various models have been developed to predict pathologic response after NAC. Several researchers proposed nomograms using preoperative clinicopathological variables (e.g. age, NAC cycles, ER, HER2, status of lymphovascular invasion) or just simple laboratory indexes (clinical tumor stage, lymphocyte to monocyte ratio, fibrinogen level, and D-dimer level) to predict pCR after NAC ${ }^{12,16}$. These nomograms were helpful in stratifying patient who would experience more or less benefit from NAC, but small patient size (less than 200) limited its validation power and result of external validation was not as good when tested in other institutions. Moreover, each nomogram consisted of different variables as researchers intended and implication of excluded or unknown covariates were neglected.

Recent studies have evaluated the significance of machine learning in classification of breast cancer. For example, Asri et al. compared different machine learning algorithms (Support Vector Machine, Decision Tree, Naive Bayes and k Nearest Neighbors) on the Wisconsin Breast Cancer datasets to assess which algorithm is the best in breast cancer risk prediction and diagnosis ${ }^{17}$. Although support vector machine was the best in terms of precision and low error rate, all four types of algorithms showed accuracy above 90\%. Delen et al. compared two data mining methods (ANN and decision trees) along with a statistical method (logistic regression) to develop a prediction model for breast cancer survivability and demonstrated that artificial neural network and decision trees performed better than logistic regression model ${ }^{18}$. These studies showed that machine-learning algorithms have promise in medical application and may provide better prediction than any other models developed before.

In this study, we compared ANN algorithm along with one of the most commonly used analytic method, nomogram, to develop a predictive model for pCR after NAC. Nomogram is a graphical tool that is designed for quick and simple determination. However, its accuracy is limited due to small number of variables that could be included in the model. Before developing a nomogram, statistical-based feature selection process must be performed to select significant factors and many potentially important clinical, radiological, or pathological factors could be dropped out during this feature reduction process. On the other hand, machine learning algorithms allow unlimited number of input variables and an optimal set of variables could be improved with training methods. Although the ANN model and nomogram were built based on the same factors for comparative purpose, we believe predictive performance of machine learning model could be improved by including extensive clinicopathologic variables. Development of a new ANN model without feature selection procedure is worthy of consideration in further study.

Nonetheless, artificial neural network has a fundamental problem regarding interpretability and lack of understanding. Despite of extreme efficiency and accuracy in dealing with large data sets, researchers are unable to explain how the algorithm uses input variables to make the output. Interpretability is crucial in

Page 13/19 
healthcare fields and inexplicability may prevent its widespread in clinical settings. Recently, researchers are trying to develop an explainable neural networks by discriminating functions the model has built on 19. Adoption of such model will certainly improve its applicability in medical fields.

To the best of our knowledge, this is the first study to compare an ANN and nomogram in prediction of PCR after NAC in breast cancer. Nomograms have provided simple and intuitive visual explanation for prediction of patients' outcome and helped clinicians in decision-making. On the other hand, machine learning algorithms are complicated in nature but their ability to learn without being explicitly programmed provide much more opportunities as data becomes complex and larger in size. Our study suggests potential of machine learning algorithms to aid clinicians in more accurate decision making for breast cancer patients.

We acknowledge that there are some limitations to our study. The main weakness is that our models were validated with internal data only. Moreover, neural network does not provide relative importance among input variables and we cannot explain which variable is more important compare with others. Lastly, machine learning has advantage in handling large size of data, but our data is limited in number. If we could further investigate using explainable neural network in large dataset, we would be able to improve its generalizability and reproducibility.

\section{Conclusions}

The comparative study of prediction models for breast cancer prognosis provide insight into the importance of machine learning algorithm in medical predictive study. The artificial neural network model demonstrated superior discrimination (AUC 0.876 [95\% Cl, 0.808-0.943]) compared with the nomogram (AUC 0.789 [95\% Cl, 0.707-0.871]). With advancement in prediction of pCR after NAC, surgeons would be able to stratify patients who would benefit from early surgical resection while non-responders to NAC may be further treated with more extensive surgery, hormonal therapy or alternative chemotherapy regimens.

\section{Declarations}

\section{Authors' contributions}

$\mathrm{JJ}$ performed study design, statistical analysis, and was a major contributor in writing the manuscript. EK and EK made significant contributions to the conception. JHK, SHK, and KJS executed neoadjuvant chemotherapy. SMK, MJ, and BLY performed radiologic examination before and after neoadjuvant chemotherapy. SYP performed histological examination of breast. HS performed study design and substantially revised the manuscript. All authors read and approved the final manuscript.

\section{Competing interests}

The authors declare that they have no competing interests. 


\section{References}

1. Schott, A. F. \& Hayes, D. F. Defining the benefits of neoadjuvant chemotherapy for breast cancer. J. Clin. Oncol. 30, 1747-1749 (2012).

2. Fisher, B. et al. Effect of preoperative chemotherapy on local-regional disease in women with operable breast cancer: Findings from national surgical adjuvant breast and bowel project B-18. J. Clin. Oncol. 15, 2483-2493 (1997).

3. Spring, L. M. et al. Pathologic Complete Response after Neoadjuvant Chemotherapy and Impact on Breast Cancer Recurrence and Survival: A Comprehensive Meta-analysis. Clin. Cancer Res. 26, 28382848 (2020).

4. Rajan, R. et al. Change in Tumor Cellularity of Breast Carcinoma after Neoadjuvant Chemotherapy As a Variable in the Pathologic Assessment of Response. Cancer 100, 1365-1373 (2004).

5. Hylton, N. M. et al. Locally advanced breast cancer: MR imaging for prediction of response to neoadjuvant chemotherapy - Results from ACRIN 6657/I-SPY TRIAL. Radiology 263, 663-672 (2012).

6. Chen, Y. et al. Estrogen receptor-related genes as an important panel of predictors for breast cancer response to neoadjuvant chemotherapy. Cancer Lett. 302, 63-68 (2011).

7. Gianni, L. et al. Gene expression profiles in paraffin-embedded core biopsy tissue predict response to chemotherapy in women with locally advanced breast cancer. J. Clin. Oncol. 23, 7265-7277 (2005).

8. Al-Azawi, D. et al. CA 15-3 is predictive of response and disease recurrence following treatment in locally advanced breast cancer. BMC Cancer 6, 3-9 (2006).

9. Rouzier, R. et al. Development and validation of nomograms for predicting residual tumor size and the probability of successful conservative surgery with neoadjuvant chemotherapy for breast cancer. Cancer 107, 1459-1466 (2006).

10. Kim, S.-Y. et al. Factors Affecting Pathologic Complete Response Following Neoadjuvant Chemotherapy in Breast Cancer: Development and Validation of a Predictive Nomogram. Radiology 203871 (2021)

11. Keam, B. et al. Nomogram predicting clinical outcomes in breast cancer patients treated with neoadjuvant chemotherapy. J. Cancer Res. Clin. Oncol. 137, 1301-1308 (2011).

12. Zhang, F. et al. A Nomogram to Predict the Pathologic Complete Response of Neoadjuvant Chemotherapy in Triple-Negative Breast Cancer Based on Simple Laboratory Indicators. Ann. Surg. Oncol. 26, 3912-3919 (2019).

13. Kourou, K., Exarchos, T. P., Exarchos, K. P., Karamouzis, M. V. \& Fotiadis, D. I. Machine learning applications in cancer prognosis and prediction. Comput. Struct. Biotechnol. J. 13, 8-17 (2015).

14. Cain, E. H. et al. Multivariate machine learning models for prediction of pathologic response to neoadjuvant therapy in breast cancer using MRI features: a study using an independent validation set. Breast Cancer Res. Treat. 173, 455-463 (2019). 
15. Chawla, N. V, Bowyer, K. W., Hall, L. O. \& Kegelmeyer, W. P. SMOTE: synthetic minority over-sampling technique. J. Artif. Intell. Res. 16, 321-357 (2002).

16. Zhang, J. et al. Can We Reliably Identify the Pathological Outcomes of Neoadjuvant Chemotherapy in Patients with Breast Cancer? Development and Validation of a Logistic Regression Nomogram Based on Preoperative Factors. Ann. Surg. Oncol. 28, 2632-2645 (2020).

17. Asri, H., Mousannif, H., Al Moatassime, H. \& Noel, T. Using Machine Learning Algorithms for Breast Cancer Risk Prediction and Diagnosis. Procedia Comput. Sci. 83, 1064-1069 (2016).

18. Delen, D., Walker, G. \& Kadam, A. Predicting breast cancer survivability: A comparison of three data mining methods. Artif. Intell. Med. 34, 113-127 (2005).

19. Vaughan, J., Sudjianto, A., Brahimi, E., Chen, J. \& Nair, V. N. Explainable neural networks based on additive index models. arXiv 1-11 (2018).

\section{Figures}

Points
ER status
HER2 status
Complete remission on MRI
Post-NAC CA15-3
Post-NAC CEA
Total Points
Linear Predictor
Probability of pCR

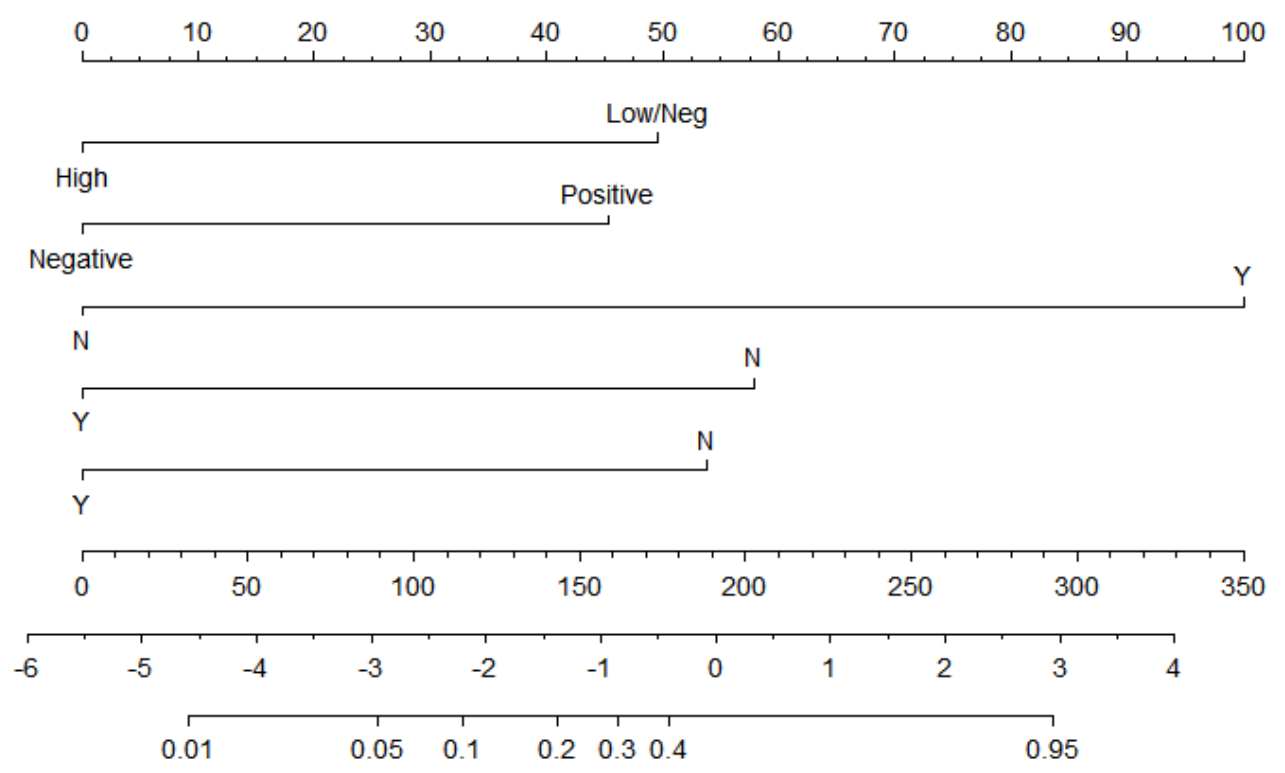

\section{Figure 1}

Nomogram model to predict pathologic complete response after neoadjuvant chemotherapy 


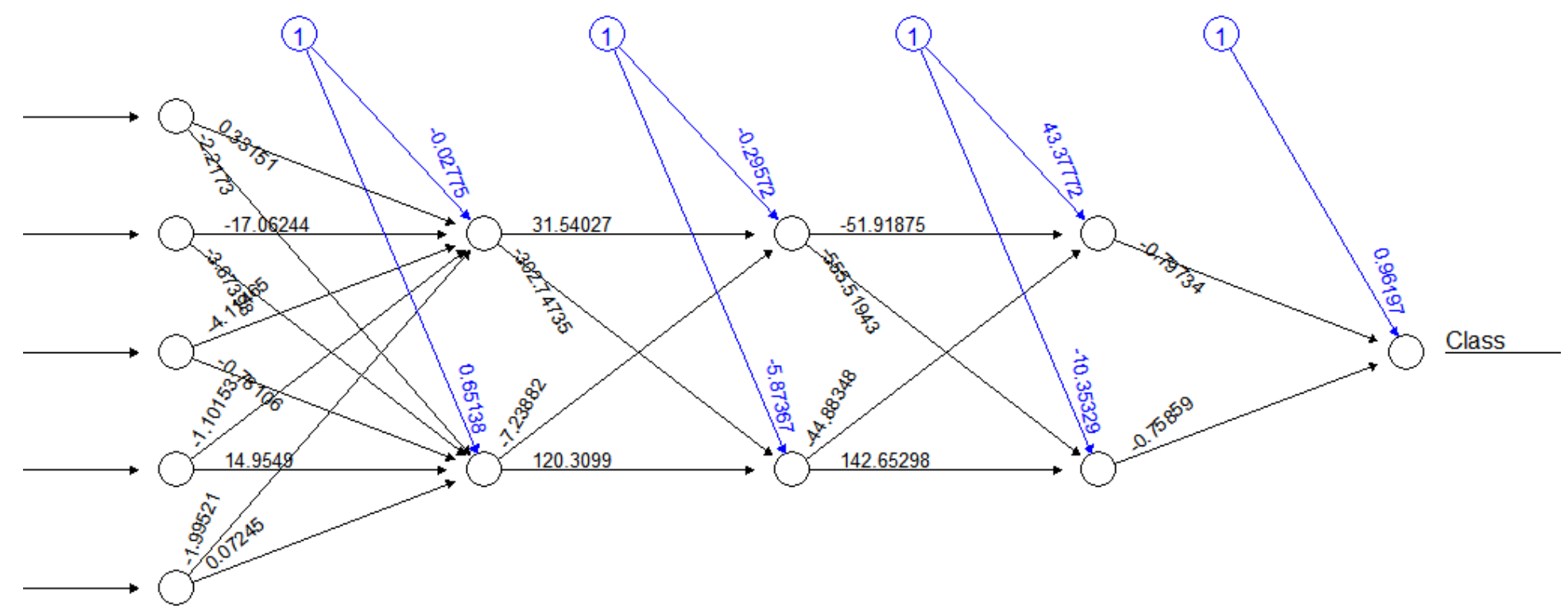

Error: 10.939991 Steps: 23105

Figure 2

Artificial neural network model to predict pathologic complete response after neoadjuvant chemotherapy 


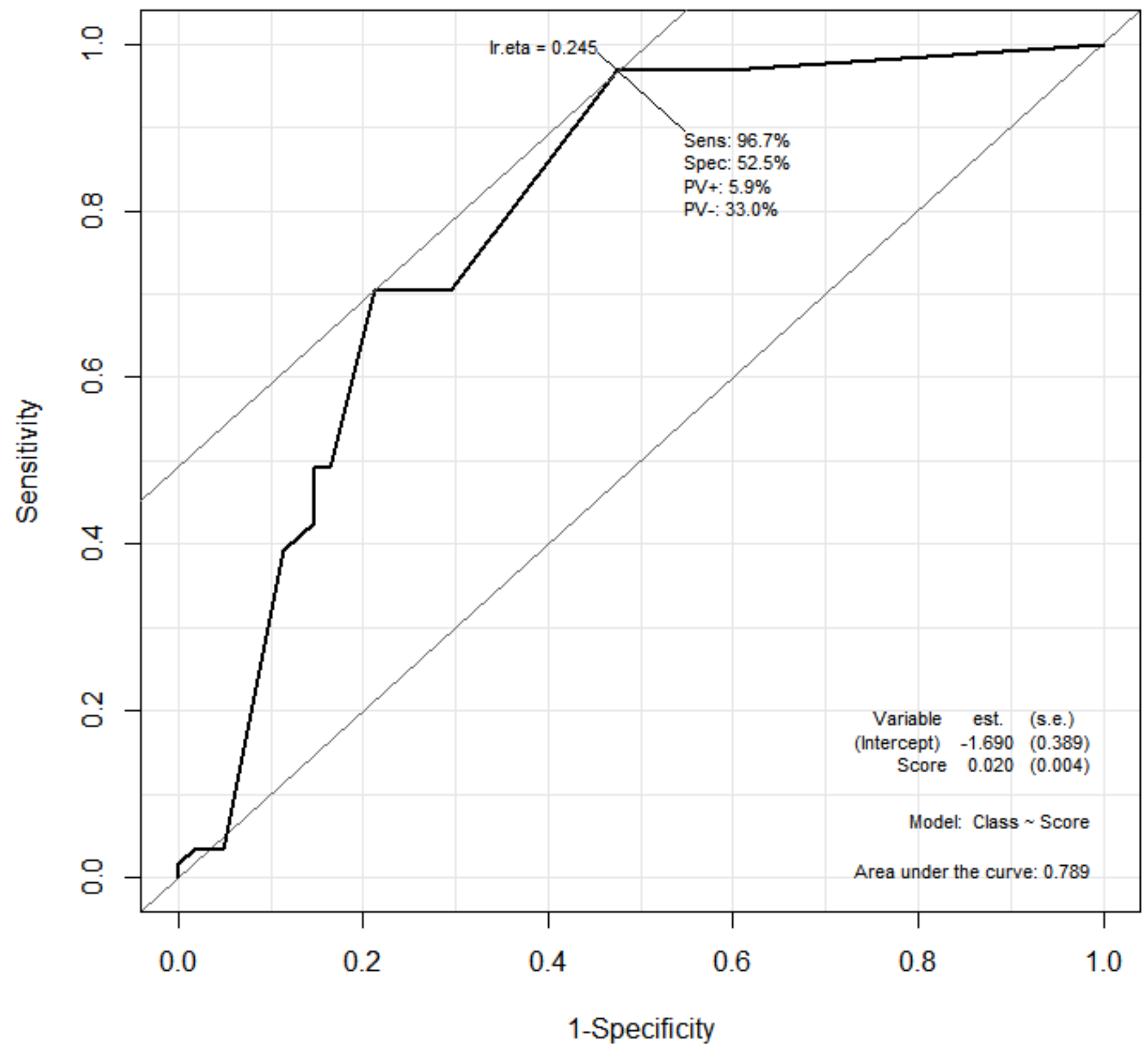

Figure 3

ROC curve showing the predictive accuracy of the nomogram 


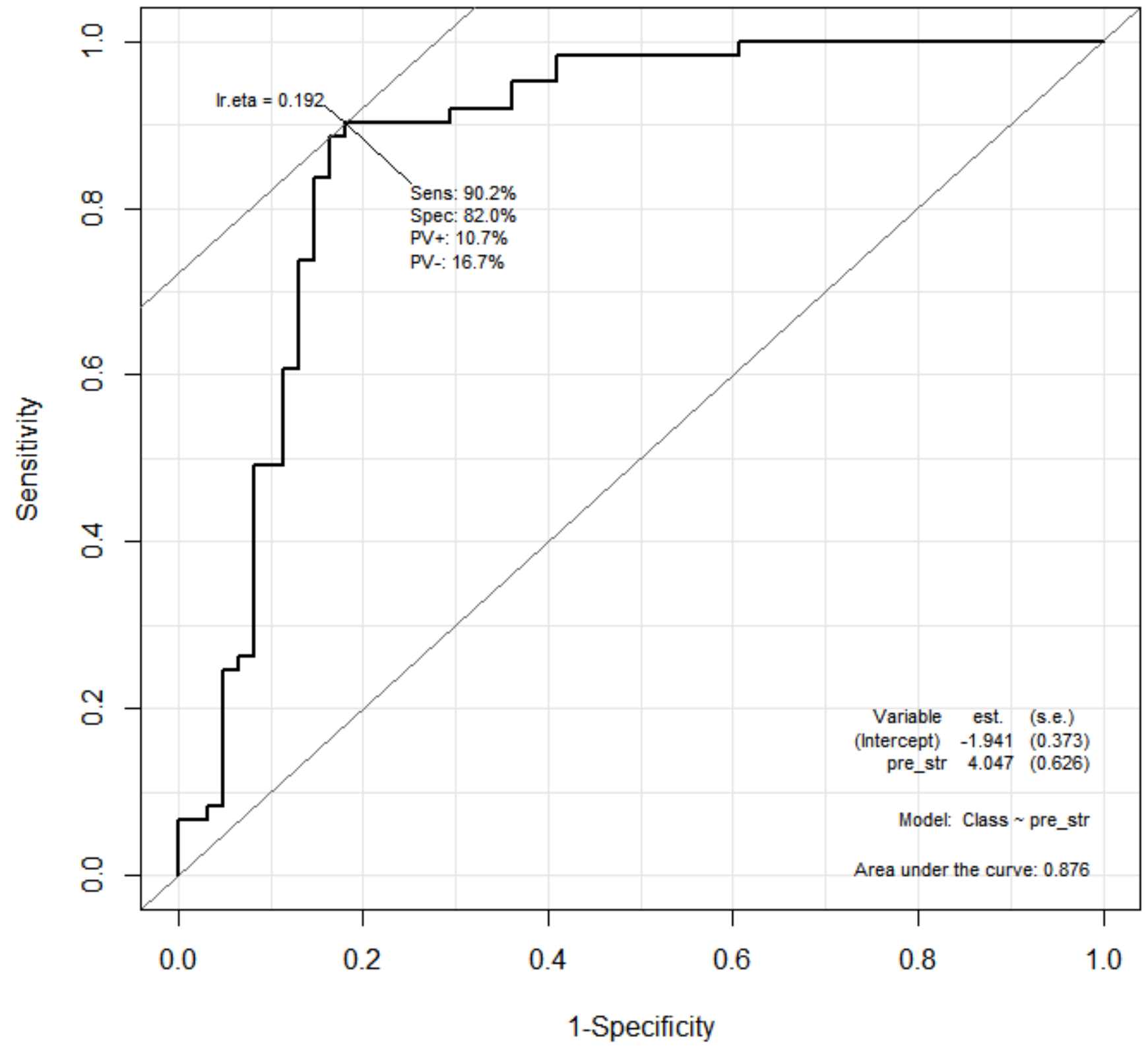

Figure 4

ROC curve showing the predictive accuracy of the artificial neural network model 